\title{
Multilocus microsatellite signature and identification of specific molecular markers for Leishmania aethiopica
}

Nigatu Kebede ${ }^{1,2,3 \dagger}$, Steve Oghumu ${ }^{2,4+}$, Alemayehu Worku ${ }^{3}$, Asrat Hailu ${ }^{5}$, Sanjay Varikuti ${ }^{2}$ and Abhay R Satoskar ${ }^{2 *}$

\begin{abstract}
Background: Leishmaniasis is a clinically and epidemiologically diverse zoonotic disease caused by obligatory, intracellular protozoan parasites of the genus Leishmania. Cutaneous leishmaniasis is the most widely distributed form of the disease characterized by skin lesions. Leishmania aethiopica is considered the predominant etiological agent in Ethiopia. The current study was aimed at developing multilocus microsatellite markers for L. aethiopica isolated from human cutaneous leishmaniasis patients in Ethiopia.
\end{abstract}

Results: L. aethiopica parasites for the study were obtained from Ethiopia and laboratory analysis was conducted at The Ohio State University. DNA was extracted from cultured parasites and an internal transcribed spacer located at the ribosomal region of L. aethiopica genomic DNA was PCR amplified for species identification. Microsatellite markers were identified using multilocus microsatellite typing. We generated an enriched genomic library, and using Primer3 software, designed PCR primers to amplify sequences flanking the detected microsatellites. Subsequent screening of the amplified markers for length variations was performed by gel electrophoresis. Using a variety of molecular methods, 22 different microsatellite markers were identified and tested for typing L. aethiopica strains using a number of clinical isolates. Of the 22 markers tested, 5 were polymorphic and showed distinctive multilocus genotypes, classifying them into four clusters. One marker was found to be specific for L. aethiopica, discriminating it from other species of Leishmania.

Conclusion: Multilocus microsatellite typing using the markers developed in this study could be useful for epidemiological and population genetic studies of strains of L. aethiopica in order to investigate the structure and dynamics of the corresponding natural foci. It could also help to answer specific clinical questions, such as the occurrence of local and diffuse lesions, strain correlates of parasite persistence after subclinical infection and lesion comparisons from patients suffering from L. aethiopica infections.

Keywords: Leishmania aethiopica, Microsatellite markers, Ethiopia

\section{Background}

Leishmaniasis is a clinically and epidemiologically diverse disease caused by obligatory, intracellular, zoonotic hemo-flagellate protozoan parasites of the genus Leishmania (family Trypanosomatidae) [1-3]. It is transmitted to humans via bites of sandflies and is prevalent in 98 countries in the world [4]. Infection by the Leishmania parasite can cause either cutaneous leishmaniasis (CL)

\footnotetext{
* Correspondence: abhay.satoskar@osumc.edu

'Equal contributors

${ }^{2}$ Department of Pathology, College of Medicine, Ohio State University

Wexner Medical Center, Columbus OH 43210, USA

Full list of author information is available at the end of the article
}

or systemic/visceral leishmaniasis (VL) [5]. CL is characterized by cutaneous lesions which develop at the site of the insect bite. Lesions can vary in severity, clinical manifestation, as well as recovery time, and in a proportion of patients, lesions can become chronic, leading to disfiguring mucosal leishmaniasis. CL can have a significant social impact as it may lead to severe stigmatization of affected individuals when lesions or scars occur on the face and exposed extremities [6]. CL is the most widely distributed form of leishmaniasis, with about one-third of the cases occurring in the Americas, the Mediterranean basin and Asia. Ten countries with the highest incidence rates are Afghanistan, Algeria,

\section{Biomed Central}


Colombia, Brazil, Iran, Syria, Ethiopia, North Sudan, Costa Rica and Peru, which together account for 70 to $75 \%$ of global estimates for CL [4]. A recent report indicates that about 20,000 - 50,000 CL cases are diagnosed each year [7]. In Ethiopia, leishmaniasis is present in both rural and urban areas and Leishmania aethiopica is considered the predominant etiological agent of CL. It causes local cutaneous leishmaniasis (LCL), mucocutaneous leishmaniasis (MCL) and diffuse cutaneous leishmaniasis (DCL) $[8,9]$. The recent increase in the number of reported CL cases in Ethiopia [10] as well as its diverse clinical manifestations highlight the epidemiological significance of the disease.

Standard diagnostic procedures for CL include detection of the parasite in a skin smear or biopsy using microscopy, or demonstration of the parasite in culture. However, even when these assays are combined, they are not sensitive enough to confirm all cases of CL. Serology is also an insufficient diagnostic tool for CL as systemic antibody responses are absent [11]. Furthermore, these techniques are unable to distinguish between different Leishmania species/strains that cause CL. Molecular techniques that detect parasite specific DNA or RNA offer definite advantages in sensitivity and speed of detection [12]. Such fast and accurate methods in the identification of disease causing parasites will further facilitate the delivery of appropriate treatment. These advantages make molecular methods a viable and attractive diagnostic strategy.

Recently, analysis of length polymorphisms of microsatellite-containing regions have become an important tool for population and genetic studies of different species [13]. Microsatellites are tandemly repeated stretches of short nucleotide motifs of 1 to 6 base pairs ubiquitously distributed in the genomes of eukaryotic organisms. They mutate at rates five to six orders of magnitude higher than that of the bulk of DNA. Microsatellite loci present high variability mainly due to allelic repeat length variations. The length variation of individual loci can easily be screened after amplification with primers that anneal specifically to their flanking regions [14]. Leishmania are relatively rich in microsatellites [15]. Multilocus microsatellite typing (MLMT) has been shown to be one of the best methods for distinction of Leishmania strains [16]. Previous population genetic studies performed by other researchers using MLMT revealed geographical and hierarchic population structures in Leishmania major, Leishmania tropica and the Leishmania donovani complex [16]. Microsatellite markers designed for species of Leishmania include 13 for L. major [17,18] 16 for L. tropica $[19,20]$ and 20 for L. donovani [17]. Two independent microsatellite loci described by Rossi et al. [15] and three genomic fragments containing several different microsatellite tracts were found to be polymorphic in Leishmania infantum [21]. However, studies identifying microsatellite markers are not available for L. aethiopica. In this current study, we describe the characterization of molecular markers for L. aethiopica isolated from human CL patients.

\section{Methods \\ L. aethiopica isolates}

L. aethiopica parasites used for this study were obtained from the Department of Immunology, Microbiology and Parasitology, School of Medicine, College of Health Sciences, Addis Ababa University (AAU), Ethiopia. Samples of $L$. aethiopica parasites and or DNA were transferred to, and maintained at the Ohio State University following institutional guidelines. L. aethiopica isolates E, B, D, $\mathrm{G}$ and $\mathrm{M}$ were obtained from CL patients in Ethiopia and were also used in this study. L. aethiopica strain MHOM/ET/1972/L102 was used as a reference strain.

\section{DNA extraction}

L. aethiopica parasites were maintained and cultured in Schneider's Drosophila medium supplemented with 20\% fetal calf serum, 1\% HEPES (4-(2-hydroxyethyl)-1piperazineethanesulfonic acid), $10 \mathrm{U}$ penicillin/ml, 100 $\mu \mathrm{g}$ streptomycin/ml and $0.05 \mathrm{mM}$ 2-mercaptoethanol. Promastigotes were harvested by centrifugation and washed twice in PBS. DNA was extracted by the method described previously [22], resuspended in Tris-EDTA buffer ( $\mathrm{pH} 7.4)$, and stored at $4^{\circ} \mathrm{C}$.

\section{L. aethiopica species identification: PCR of ITS region and sequencing}

The internal transcribed spacer in the ribosomal region was amplified with primers ITSFW (5' -ACACTCAGG TCTGTAAAC) and ITSRV (5'-CTGGATCATTTTCC GATG) as described previously [23]. In brief, a total of $10 \mathrm{ng}$ template DNA in $2 \mu \mathrm{l}$ was added to the reaction mixture containing $20 \mathrm{pmol}$ of each primer in $5 \mu \mathrm{l}, 12.5$ $\mu \mathrm{l}$ Taq polymerase (Perkin-Elmer-Cetus, Norwalk, CT, USA) and $5.5 \mu \mathrm{l}$ distilled water. PCR cycling conditions were as follows: initial denaturation of $95^{\circ} \mathrm{C}$ for $5 \mathrm{~min}$, 35 cycles of $95^{\circ} \mathrm{C}$ for $20 \mathrm{~s}, 50^{\circ} \mathrm{C}$ for $30 \mathrm{~s}, 72^{\circ} \mathrm{C}$ for $1 \mathrm{~min}$ followed by a final extension of $72^{\circ} \mathrm{C}$ for $6 \mathrm{~min}$. PCR products containing the amplified ITS region were separated in a $1 \%$ agarose gel with $1 \times$ TAE buffer and visualized under a UV transilluminator. Desired bands of expected sizes were excised and extracted using a Qiagen gel extraction kit (Valencia, CA, USA) according to the manufacturers' instructions. Samples were submitted to the Plant-Microbe Genomics Facility at the OSU for sequencing. Sequence alignments were performed using the NIH based Basic Local Alignment Search Tool (BLAST) for identification of Leishmania species. 


\section{Microsatellite library enrichment}

We constructed an enriched genomic library based on the protocols developed by Bloor et al., [24]. Ten micrograms of genomic DNA extracted from L. aethiopica was digested with HaeIII. The fragments were ligated to specific adaptors consisting of "Oligo A" (5'-GGC CAG AGA CCC CAA GCT TCG-3') and "Oligo B" (5' $\mathrm{PO}_{4-}$ GAT CCG AAG CTT GGG GTC TCT GGC C-3”) [25]. Fragments between 400 and $800 \mathrm{bp}$ were excised from the agarose gel, extracted, and concentrated using YM50 spin columns. The fractionated DNA was denatured and hybridized at a temperature of $55^{\circ} \mathrm{C}$ to $(\mathrm{GT})_{10} 3^{\prime}$ biotinylated oligonucleotides bound to M-280 streptavidin-coated magnetic beads. After incubation at $72^{\circ} \mathrm{C}$ for $2 \mathrm{~h}$, unbound DNA and excess oligonucleotides were removed following differential stringency washes. PCR amplification of the immobilized fragments was conducted by using a suspension of $1 \mathrm{mg}$ magnetic beads containing enriched DNA fragments as template and 30 pmol of "Oligo A" primer. The terminal elongation step was extended to $30 \mathrm{~min}$. The amplified fragments were ligated to TOPO vector and transformed into competent E. coli, according to the manufacturer's instructions (Life Technologies, Grand Island, NY). Cells were plated out on LB agar plates and incubated for overnight at $37^{\circ} \mathrm{C}$. For screening of the library, colony PCRs were conducted by using a $\mathrm{T}_{\mathrm{A}}$ of $55^{\circ} \mathrm{C}, 10$ pmol of primers oligo $A$ and $(\mathrm{GT})_{10}$. Microsatellite-containing fragments produced double bands in subsequent gel electrophoresis. Plasmid DNAs from positive clones were sequenced at the Plant-Microbe Genomics Facility at the Ohio State University, to confirm the presence of microsatellites.

\section{Design of microsatellite markers}

Using Primer3 software [26], PCR primers between 18 bp and $22 \mathrm{bp}$ in length were designed to amplify sequences flanking the detected microsatellites. Primers were chosen from sequences 1 to 25 nucleotides upstream and downstream of the microsatellite repeat. A BLAST search was conducted for all markers to find corresponding sequences within the Leishmania genome to determine the chromosomes on which the amplified regions were localized.

\section{Analysis of microsatellite variation}

Amplification reactions were performed using $40 \mathrm{ng}$ Leishmania genomic DNA as template and 10 pmol of each primer. PCR products were screened for length variations by agarose gel electrophoresis in $4 \%$ MetaPhor agarose gels (Lonza Rockland, ME, USA), prepared according to the manufacturer's instructions.

\section{Ethical considerations}

This study was reviewed and approved by the Institutional Review Board (IRB) of the School of Public Health, AAU. A Material Transfer Agreement (MTA) was signed between AAU and OSU.

\section{Results}

Identification of $L$. aethiopica from clinical isolates

Earlier work identified primers for identification of $L$. aethiopica from clinical isolates [25]. We were unable to differentiate L. aethiopica from other species of Leishmania (L. tropica, L. major, L. mexicana, L. donovani and $L$. infantum) with the use of these previously validated L. aethiopica specific primers (Figure 1A). On the other hand, although PCR analysis of the ITS1 region was unable to yield distinguishable bands using the Leishmania species used, sequencing of the PCR amplified ITS1 region and subsequent BLAST searches on genome databases identified L. aethiopica isolates. ITS1 sequences from L. aethiopica isolates used in this study were $99 \%$ homologous to L. aethiopica strains and shared $90 \%$ homology to L. tropica, and $83 \%$ to L. major sequences in genome databases (Figure 1B). We therefore utilized ITS1 gene sequencing for identification of all L. aethiopica isolates used in this study.

\section{Frequency and distribution of GT rich microsatellites}

Previous methods developed for analysis of strain variation within $L$. aethiopica have not been very successful. We therefore examined whether the L. aethiopica genome was rich in microsatellites and determined the potential for utilizing microsatellites as a basis for discriminating between isolates of L. aethiopica. Other species of Leishmania have been shown to be rich in microsatellites and GT rich microsatellite markers have been identified for L. major [17], L. tropica [19] and $L$. donovani [19]. We therefore analyzed the frequency and distribution of GT rich microsatellites in L. aethiopica isolates. Following microsatellite enrichment, a total of 92 clones presumably containing GT rich microsatellite structures were obtained from the L. aethiopica $(\mathrm{GT})_{10}$ enriched genomic library. Colony PCR of enriched clones using oligo A primers revealed a significant number of regions which were positive for GT containing sequences as shown by the presence of double bands after separation of PCR products on a $2 \%$ agarose gel (Figure 2). Subsequent DNA sequencing confirmed the presence of GT containing sequences in about 22 of these regions. While some of these sequences were homologous to other Leishmania species and could be mapped to specific chromosomes based on sequence alignment searches, other sequences seemed to be unique to L. aethiopica (Table 1). 


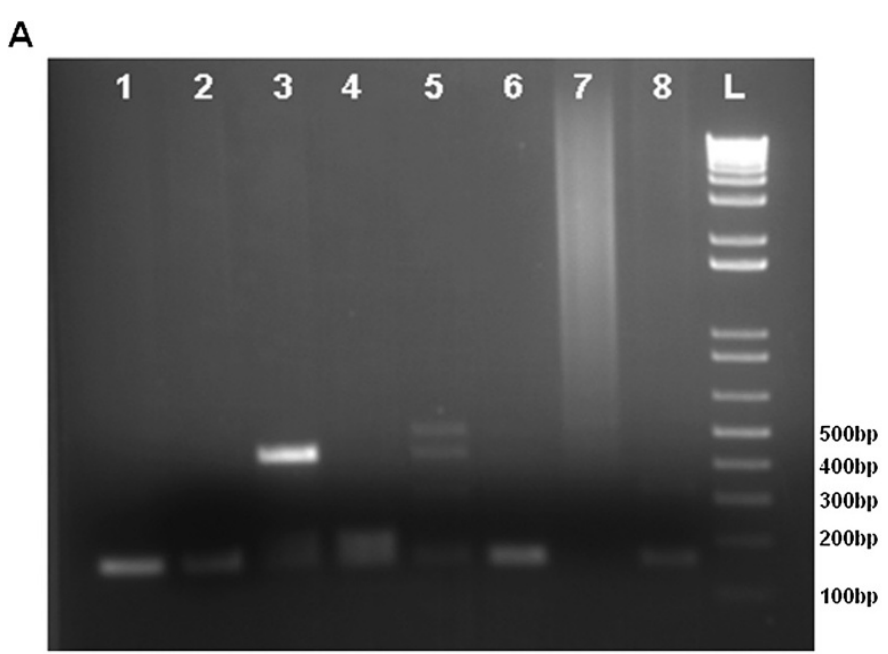

\section{B}

\begin{tabular}{|c|c|}
\hline $\begin{array}{l}\text { ference: } \\
\text { aethiopica: } \\
\text { major: } \\
\text { tropica: }\end{array}$ & 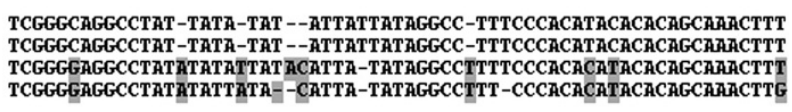 \\
\hline $\begin{array}{l}\text { eference: } \\
\text { aethiopica: } \\
\text {. major: } \\
\text { tropica: }\end{array}$ & \\
\hline $\begin{array}{l}\text { Reference: } \\
\text { L. aethiopica: } \\
\text { L. major: } \\
\text { L. tropica: }\end{array}$ & 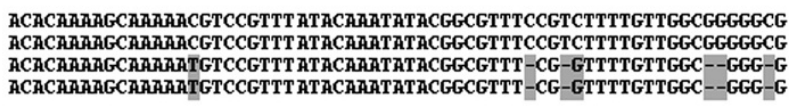 \\
\hline tropica: & $\begin{array}{l}\text { GTGCGTGTGTGTGGATAACGGCTCACATAARCGTGTCGCGATGGATGACTTGGCTTCCTATTT } \\
\text { G--CGTGTGTGTGGATAACGGCTCACATARCGTGTGGGATGGATGACTTGGCTTCCTATTT } \\
\text { G--CGTGTGTGTGGATAACGGCTCACATAACGTGTCGCGATGGATGACTTGGCTTCCTATTT }\end{array}$ \\
\hline
\end{tabular}

Figure 1 Identification of L. aethiopica from clinical isolates. A) PCR amplification of Leishmania genomic DNA from different species using previously developed primers MATRAE2 and Ae2.1 subjected to electrophoresis on a 1\% agarose gel. Lanes 1 and 2 are PCR products from 2 different $L$. aethiopica isolates used in the study. Lanes $3,4,5,6,7$ and 8 are PCR products amplified from genomic DNA of L. donovani, L. major, L. mexicana, L. tropica, L. infantum and mouse, respectively. $L$ is $1 \mathrm{~kb}$ DNA ladder. B) Basic Local Alignment search of sequences derived from PCR amplification of the ITS1 region of L aethiopica, L major and L. tropica, using L5.8S/LISTR primers. Genomic DNA from L. aethiopica MHOM/ET/ 1972/L102 was used as a reference strain.

\section{Identification of strains of L. aethiopica based on} microsatellite typing

To determine whether polymorphisms in the GT containing microsatellites exist within strains of $L$. aethiopica, we designed primers flanking each of the 22 identified microsatellite sequences (Table 1). Primers were developed to align closely to the CA/GT repeats to reduce bias due to additional insertion/deletion events in the flanking regions. Furthermore, fragment length analysis in a MetaPhor agarose gel was used to elucidate the resulting short PCR products.

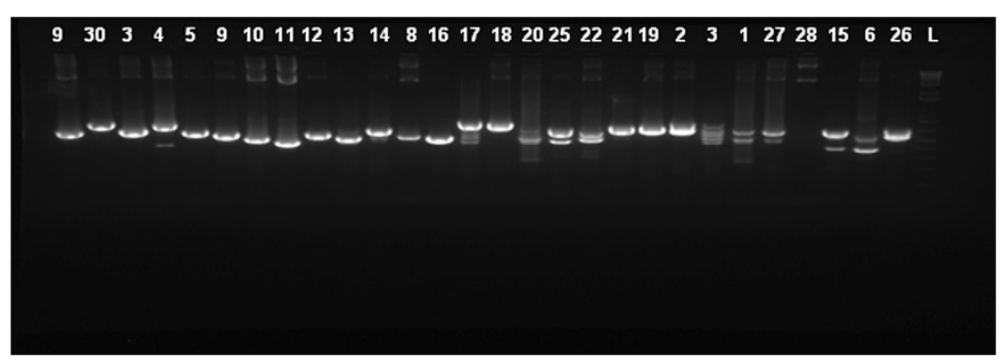

Figure 2 Frequency and distribution of GT rich microsatellites. PCR screening of select microsatellite containing E. coli clones using Oligo A and $\mathrm{GT}_{10}$ primers and transformed E. coli plasmid DNA as template. PCR products were electrophoresed on a $2 \%$ agarose gel. Clones that contained a microsatellite demonstrated a double band PCR product. 
Table 1 Microsatellite markers of $L$. aethiopica developed and tested in this study

\begin{tabular}{|c|c|c|c|c|c|}
\hline $\mathrm{CO}$ & REPEAT ARRAY & $\begin{array}{l}\text { FORWARD PRIMER SEQUENCE } \\
\qquad\left(5^{\prime}-3^{\prime}\right)\end{array}$ & $\begin{array}{l}\text { REVERSE PRIMER SEQUENCE } \\
\left(5^{\prime}-3^{\prime}\right)\end{array}$ & CHROMOSOME LOCATION & POLYMORPHISM \\
\hline 1 & (CA) 10 & TGAACAGTACGTCCAGGAGGA & GTGGTGGAAGAGGGAGAGAG & NA & $P$ \\
\hline 3 & $(\mathrm{GT}) 20$ & TCTTGCTTACGCGTGGACTA & ACGAGGCAGGAGGGAGAG & Lmj11 & NA \\
\hline 4 & $(C A) 12$ & TGCGCCAGTTTCTATGACTG & TGTGTITGTGTGCGTGGATA & Lm×32, Ld33 & $P$ \\
\hline 5 & (GT)10 & TTATGATGTAGGCAGGGTAGAGT & GTATTGGGCAAAGGGACCTT & Lmj31, Ld31 & NA \\
\hline 6 & $(C A) 29$ & TGCTCATGGTGGATGTGC & GTCGTGTGGTCGTGTATGTG & Lmj31 & $P$ \\
\hline 7 & $(C A) 12$ & TTCGTCGCTCTTGTCTCTTG & TAGTCCACGCGTAAGCAAGA & Lmj24 & NA \\
\hline 8 & (CA) 15 & TCССTСTCTTCСTCTGTTCC & CAGATGCGCAAGAATCAAAA & Lmj, Ld15 & NA \\
\hline 11 & $(C A) 16$ & СTCССTCTCTTCСTCTGTTCC & TCTCTCGGTGAGCCAGATG & Lmj, Ld15 & NA \\
\hline 13 & $(\mathrm{GT}) 16$ & GGTCGTTGTGACGCCAGT & ACTGCGTTCTGTGTGTCTCCT & Lmj31 & NA \\
\hline 15 & (GT) 10 & CCACCACCACACTITTTCGT & GATACCGGCTGATGATGAGG & NA & NA \\
\hline 16 & (GT)13 & TCTTGCCTCTITAATCTGTGTCC & GTTGTGGTGGTTGATCCGTT & Lmj34 & NA \\
\hline 17 & $(C A) 28$ & CTCACACATGGATGCAAAGC & CGTGTGTGTGTGCCATACAA & NA & NA \\
\hline 18 & $(\mathrm{GT}) 21$ & GGAGGGGGAGGAGTCTAAAA & TGTACTGCCGAGATTGAACG & Lmj34, Li31,34, Ld8, 34 & NA \\
\hline 19 & (GT)50, 30 & TCTTGCTTACGCGTGGACTA & TAGAGTTGATTGCACGTGGC & Lmj12 & NA \\
\hline 20 & (GT) 11 & TACTTCGCGCACCGTCTCT & TTGTCGGTCGTGACTACTGC & Lmj26 & NA \\
\hline 21 & (GT) 20 & GCTTACGCGTGGACTACCA & CTATCGAGGGTCGGCAAC & Lmj11,26 & NA \\
\hline 22 & (GT)16 & GGCGCGATATCCACTTCTT & TGTAAGTGTGTGGGTGGGTG & Lmj18, Lin18 & $P$ \\
\hline 23 & (GT)12 & GTGGGTTITTGAATTATGTCGTC & GTATTGGGCAAAGGGACCTT & Lmj31, Ld31, Lin31, Lm×30 & NA \\
\hline 26 & (GT)15 & TCTTGCTTACGCGTGGACTA & TAGAGTTGATTGCACGTGGC & Lmj12, Ld12 & NA \\
\hline 27 & (GT)40 & CGTACGTCTGTTGCCTCTGT & CACTACTCCGCAGAACCGTA & Ld26 & $P$ \\
\hline 28 & (GT)13 & CTCTTGCTTACGCGTGGACT & AGAGACAAGAGCGACGAAGC & Lmj24 & NA \\
\hline 30 & (GT)16 & CCTITGCCTTCTCCCTTTT & GAGCTTGGCGTAATCATGGT & NA & NA \\
\hline
\end{tabular}

Note: Lmj: Leishmania major, Lmx: L. mexicana, Lin: L. infantum, Ld: L. donovani. NA: not available.

All of the 22 microsatellite markers were tested with the different isolates of two previously identified strains of $L$. aethiopica in Ethiopia. The size of the amplified fragments (and thus the number of repeats) was compared to the size of fragments from cloned L. aethiopica strain $\mathrm{MHOM} / \mathrm{ET} / 1972 / \mathrm{L} 102$. Five of these markers demonstrated polymorphisms, as shown by variable PCR product sizes, within the species and strains of $L$. aethiopica (Figure 3). Microsatellite typing using these 5 markers further subdivided 2 previously identified strains of L. aethiopica into 4 groups. Two isolates (D and G) shared the same GT repeat profile while the other isolates were different based on their PCR product sizes.

Identification of species specific primers for L. aethiopica Specie identification of clinical isolates from patients in Ethiopia has presented challenges. A number of PCR primers have been developed for identification of $L$. aethiopica [27], but we were unable to detect L. aethiopica specific bands using these primers (Figure 1A). We therefore tested PCR primers pairs which we had previously observed to be specific for L. aethiopica isolates. Our analysis revealed that PCR detection using primer 15 is specific for L. aethiopica. The specificity of this marker was evaluated using DNA extracted from different Leishmania species as well as from clinical isolates. DNA isolated from each species of L. aethiopica showed a $100 \mathrm{bp}$ product after PCR amplification using primer 15, while DNA isolated from other species of Leishmania gave no PCR product under the same PCR conditions used (Figure 4). A PCR assay using this new primer could potentially be utilized as a tool for the diagnosis of CL due to L. aethiopica in patients.

\section{Discussion}

In this study, we were able to identify hypervariable microsatellite loci and compile a set of markers usable for future epidemiological and population genetic studies for strains of L. aethiopica. Since this method is rapid and reproducible, we believe that it can be used for the reliable identification and characterization of $L$. aethiopica parasites. Of the 22 markers developed in this study, 5 polymorphic markers and one species specific marker were identified. MLMT has an advantage over other molecular techniques as results are reproducible and exchangeable between laboratories [16]. It has 

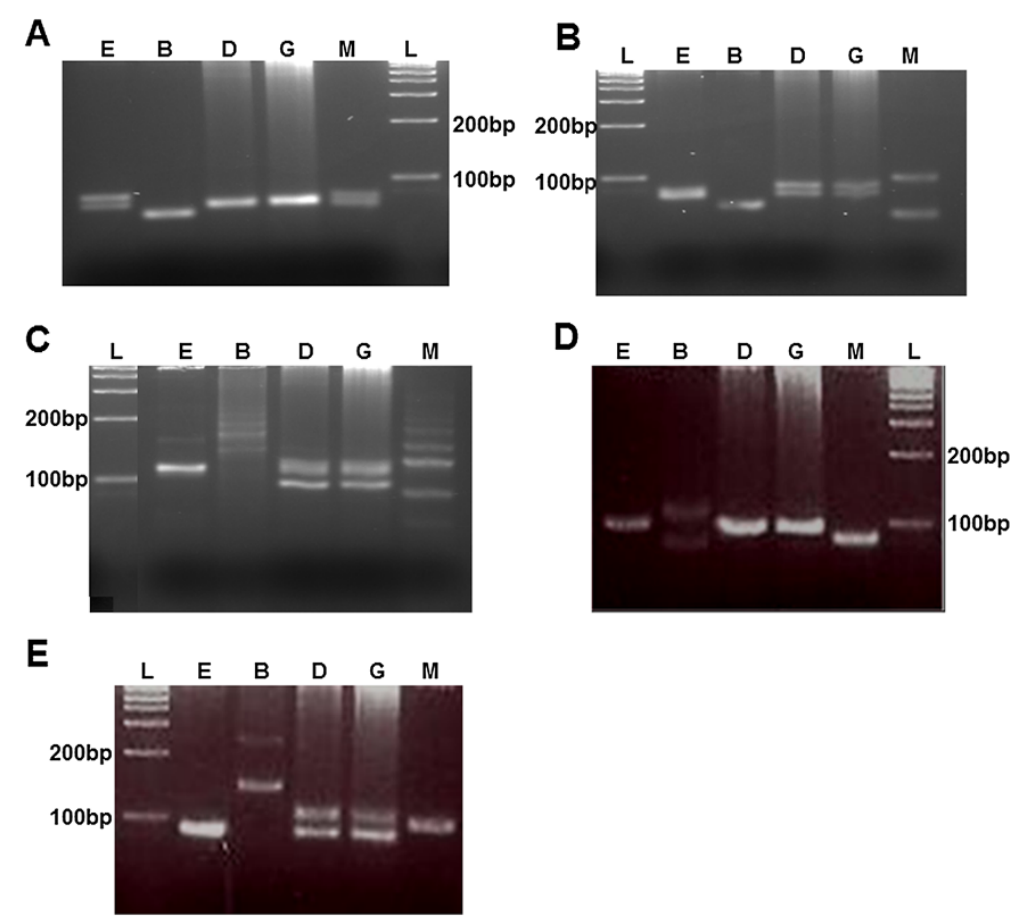

Figure 3 Identification of strains of $L$. aethiopica based on microsatellite typing. PCR analysis of genomic DNA extracted from various isolates of L. aethiopica using various markers designed from microsatellite regions identified in this study. PCR products were electrophoresed on a $4 \%$ Metaphor agarose gel and analyzed for fragment length variation. E, B, D, G and M represent different isolates of $L$ aethiopica used in this study. A) Marker 1, B) Marker 4, C) Marker 6, D) Marker 22 E) Marker 27. L is 1 kb ladder.

proved to be a powerful tool for population genetic investigations, as well as epidemiological investigations, of Leishmania species [21]. These short sequence repeats are highly polymorphic, codominant, and dispersed throughout the parasite genome. It has been shown that microsatellite loci of the family Trypanosomatidae are stable under laboratory conditions and can be detected directly in biological samples containing low amounts of parasitic DNA [21,28]. In addition, the results of microsatellite analysis are much easier to compare between laboratories and store in databases [29].

This present study using MLMT divided the available isolates of L. aethiopica into four clusters. Previous studies conducted using L. aethiopica isolated from the skin of patients indicated genetic variation within the species; Multilocus Enzyme Electrophoresis (MLEE) separated strains into two different genetic groups [23]. However, the techniques used suffer from poor reproducibility

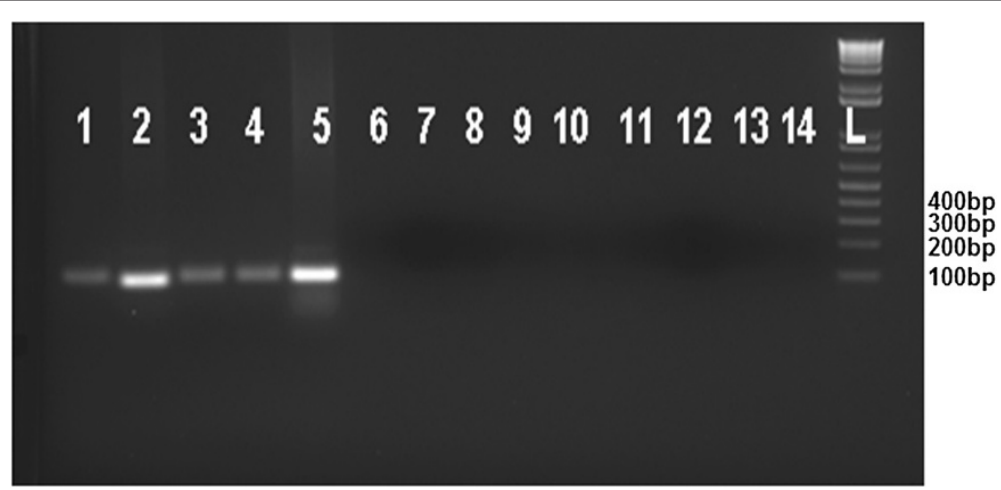

Figure 4 Identification of species specific primers for L. aethiopica. Multilocus microsatellite markers developed in the study analyzed for Leishmania species specificity. PCR analysis of genomic DNA extracted from different Leishmania species using primer 15 with an annealing temperature of $55.5^{\circ} \mathrm{C}$. PCR products were electrophoresed on a $2 \%$ agarose gel and analyzed for the presence of a 100 bp band. Lanes: 1-5: different isolates of L. aethopica; 6: L. donovani; 7: L. major; 8: L. mexicana; 9-13:L. tropica; 14: L infantum. L represents 1 kb ladder. 
[29]. In addition polymorphic repeats are not conserved between different species of Leishmania [17]. Recently, analysis of length polymorphisms of microsatellitecontaining regions has become an important tool for population and genetic studies for many different species $[13,30]$. Microsatellites are tandemly repeated stretches of short nucleotide motifs which mutate at rates of five to six orders of magnitude higher than that of the bulk of DNA and present high variability mainly due to allelic repeat length variation. The length variation of individual loci can easily be screened after amplification with primers that anneal specifically to their flanking regions [14]. These microsatellites are used for mapping genes in the genome because of their abundant distribution [31,32]

A number of researchers have developed microsatellite markers for different species of Leishmania including L. major [17,18], L. tropica [19,20] and L. donovani [17], as well as other organisms such as Penicillium marneffei [33], which are now available and used for MLMT. To our knowledge, this is the first report demonstrating the use of MLMT for the molecular characterization of L. aethiopica, a parasite which is highly prevalent in Ethiopia and the major cause of LCL, MCL and DCL, accounting for an annual incidence of 50,000 cases [4].

Feasibility of high-throughput MLMT requires the optimization of PCR product analysis. In this study we show that MetaPhor gel electrophoresis and sequencing both produced analogous and reproducible results. Sequencing was used to determine the number of repeats. This is indispensable for the analysis of large fragments containing more than one microsatellite. However, this method is expensive and sequences containing small tandem repeats may be difficult to process. We demonstrate in this study that using MetaPhor agarose gel electrophoresis to screen for polymorphisms produces sufficient resolution to distinguish between $L$. aethiopica strains and could identify short tandem repeats [29].

All of the L. aethiopica isolates tested showed exclusive multilocus microsatellite patterns using the five identified markers. Thus MLMT could potentially enable researchers to potentially track strains of this parasite, making this an effective epidemiological tool. Even with these promising results, however, more isolates will need to be processed to confirm the spatial clusters or subdivisions of L. aethiopica in Ethiopia. Studies conducted on $L$. aethiopica are few compared to other species of Leishmania possibly due to its prevalence restricted to the eastern part of Africa. This study provides tools that will enable further molecular epidemiological and population genetic research on CL caused by L. aethiopica.

Primers previously developed for L. aethiopica were unable to identify the different Leishmania species and isolates in our study. We therefore utilized the previously characterized ITS1 regions to identify and confirm the identity of the Leishmania species used in this study. Sequencing of PCR products generated using ITS1 specific primers and performing sequence alignments against the Leishmania genome database enabled us to identify these isolates. The species specific primers developed in our current study could provide a quicker, cost effective and highly useful tool for the typing/diagnosis of $L$. aethiopica on clinical samples. This would be useful for case detection, determination of appropriate therapeutic regimens as well as implementation of control measures. Further, since this method does not require a restriction enzyme digestion step as in restriction fragment length polymorphism (RFLP), it provides an added advantage in accelerating species identification.

\section{Conclusion}

In conclusion, we demonstrate in this study, the successful development of markers for multilocus microsatellite typing of strains of $L$. aethiopica. We further successfully designed a species specific marker for L. aethiopica. The MLMT markers developed in this study have great potential for use in epidemiological and population genetic studies of strains of L. aethiopica. It will potentially facilitate investigation of the structure and dynamics of the corresponding natural foci. It will also help to answer specific clinical questions, such as the occurrence of local and diffuse lesions, strain correlates of parasite persistence after subclinical infection, lesion comparisons from patients suffering from L. aethiopica infections and the determination of endogenous and/or exogenous reinfection associated with immunosuppression.

\section{Competing interest}

The authors declared that they have no competing interest.

\section{Authors' contributions}

NK, SO, ARS, AW, AH conceived the study. NK, SO, ARS designed the experiments. NK, SO, SV performed the experiments. NK, SO, ARS, AW, AH analyzed the data. NK, SO, ARS prepared the paper. All authors contributed to the writing of the manuscript and approved the submitted version of the manuscript.

\section{Acknowledgements}

The authors are grateful to One Health, PHPID program (awarded to NK), the Department of Pathology, Ohio State University, the Nathan Cummings Foundation, as well as the National Institute of Dental \& Craniofacial Research Training grant (awarded to SO) for providing the scholarship and covering the expenses of the study. ALIPB and SPH of AAU are also acknowledged.

\section{Financial support}

This study was supported by the Public Health Preparedness for Infectious Diseases (PHPID) program at The Ohio State University, and the National Institute of Dental \& Craniofacial Research Training grant T32DE014320 (awarded to SO)

\section{Author details}

${ }^{1}$ Aklilu Lemma Institute of Pathobiology, Addis Ababa University, Addis Ababa, Ethiopia. ${ }^{2}$ Department of Pathology, College of Medicine, Ohio State University Wexner Medical Center, Columbus OH 43210, USA. ${ }^{3}$ Department of Epidemiology and Biostatistics, School of Public Health, Addis Ababa 
University, Addis Ababa, Ethiopia. ${ }^{4}$ Department of Oral Biology, College of Dentistry, Ohio State University, Columbus OH 43210, USA. ${ }^{5}$ Department of Microbiology, Immunology and Parasitology, School of Medicine, Addis Ababa University, Addis Ababa, Ethiopia.

Received: 22 February 2013 Accepted: 24 May 2013

Published: 4 June 2013

\section{References}

1. Ashford RW: The leishmaniases as model zoonoses. Ann Trop Med Parasitol 1997, 91(7):693-701.

2. Bañuls AL, Hide M, Prugnolle F: Leishmania and the leishmaniases: a parasite genetic update and advances in taxonomy, epidemiology and pathogenicity in humans. Adv Parasitol 2007, 64:1-109.

3. Singh S: New developments in diagnosis of leishmaniasis. Indian J Med Res 2006, 123(3):311-330

4. Alvar J, Vélez ID, Bern C, Herrero M, Desjeux P, Cano J, Jannin J, Den Boer M, Team WLC: Leishmaniasis worldwide and global estimates of its incidence. PloS One 2012, 7(5):e35671.

5. Murray HW, Berman JD, Davies CR, Saravia NG: Advances in leishmaniasis. Lancet 2005, 366(9496):1561-1577.

6. Who: Control of the leishmaniases. In Report of a meeting of the WHO Expert Committee on the Control of Leishmaniases, Geneva, 22-26 March 2010. Geneva: WHO; 2010.

7. Lemma W, Erenso G, Gadisa E, Balkew M, Gebre-Michael T, Hailu A: A zoonotic focus of cutaneous leishmaniasis in Addis Ababa, Ethiopia. Parasites \& Vectors 2009, 2(1):60

8. Ashford RW, Bray MA, Hutchinson MP, Bray RS: The epidemiology of cutaneous leishmaniasis in Ethiopia. Trans R Soc Trop Med Hyg 1973, 67(4):568-601

9. Gebre-Michael T, Balkew M, Ali A, Ludovisi A, Gramiccia M: The isolation of Leishmania tropica and L. aethiopica from Phlebotomus (Paraphlebotomus) species (Diptera: Psychodidae) in the Awash Valley, northeastern Ethiopia. Trans R Soc Trop Med Hyg 2004, 98(1):64-70.

10. Negera E, Gadisa E, Yamuah L, Engers H, Hussein J, Kuru T, Hailu A, Gedamu $L$, Aseffa A: Outbreak of cutaneous leishmaniasis in Silti woreda, Ethiopia: risk factor assessment and causative agent identification. Trans $R$ Soc Trop Med Hyg 2008, 102(9):883-890.

11. Herwaldt BL: Leishmaniasis. Lancet 1999, 354(9185):1191-1199.

12. Wilson SM: DNA-based methods in the detection of Leishmania parasites: field applications and practicalities. Ann Trop Med Parasitol 1995, 89(Suppl 1):95-100.

13. Tóth G, Gáspári Z, Jurka J: Microsatellites in different eukaryotic genomes: survey and analysis. Genome Res 2000, 10(7):967-981.

14. Sampaio P, Gusmão L, Alves C, Pina-Vaz C, Amorim A, Pais C: Highly polymorphic microsatellite for identification of Candida albicans strains. J Clin Microbiol 2003, 41(2):552-557.

15. Rossi V, Wincker P, Ravel C, Blaineau C, Pagés M, Bastien P: Structural organisation of microsatellite families in the Leishmania genome and polymorphisms at two (CA)n loci. Mol Biochem Parasitol 1994, 65(2):271-282.

16. Schönian G, Kuhls K, Mauricio IL: Molecular approaches for a better understanding of the epidemiology and population genetics of Leishmania. Parasitology 2011, 138(4):405-425.

17. Jamjoom MB, Ashford RW, Bates PA, Kemp SJ, Noyes HA: Towards a standard battery of microsatellite markers for the analysis of the Leishmania donovani complex. Ann Trop Med Parasitol 2002, 96(3):265-270.

18. Hamarsheh O: Distribution of Leishmania major zymodemes in relation to populations of Phlebotomus papatasi sand flies. Parasites \& Vectors 2011, 4(1):9.

19. Schwenkenbecher JM, Fröhlich C, Gehre F, Schnur LF, Schönian G: Evolution and conservation of microsatellite markers for Leishmania tropica. Infect Genet Evol 2004, 4(2):99-105.

20. Azmi K, Schnur L, Schonian G, Nasereddin A, Pratlong F, El Baidouri F, Rave C, Dedet J-P, Ereqat S, Abdeen Z: Genetic, serological and biochemical characterization of Leishmania tropica from foci in northern Palestine and discovery of zymodeme MON-307. Parasites \& Vectors 2012, 5(1):121.

21. Bulle B, Millon L, Bart JM, Gállego M, Gambarelli F, Portús M, Schnur L, Jaffe $\mathrm{CL}$, Fernandez-Barredo S, Alunda JM, et al: Practical approach for typing strains of Leishmania infantum by microsatellite analysis. J Clin Microbiol 2002, 40(9):3391-3397.

22. Meredith SE, Zijlstra EE, Schoone GJ, Kroon CC, Van Eys GJ, Schaeffer KU, El-Hassan AM, Lawyer PG: Development and application of the polymerase chain reaction for the detection and identification of Leishmania parasites in clinical materia. Arch Inst Pasteur Tunis 1993, 70(3-4):419-431

23. Schönian G, Akuffo H, Lewin S, Maasho K, Nylén S, Pratlong F, Eisenberger $C L$, Schnur LF, Presber W: Genetic variability within the species Leishmania aethiopica does not correlate with clinical variations of cutaneous leishmaniasis. Mol Biochem Parasitol 2000, 106(2):239-248.

24. Bloor FSB PA, Watts PC, Noyes HA, Kemp SJ: Microsatellite Libraries by Enrichment, Manual. University of Liverpool, School of Biological Sciences; 2001. http://www.genomics.liv.ac.uk/animal/MICROSAT.PDF

25. Refseth UH, Fangan BM, Jakobsen KS: Hybridization capture of microsatellites directly from genomic DNA. Electrophoresis 1997, 18(9):1519-1523.

26. Rozen S, Skaletsky H: Primer3 on the WWW for general users and for biologist programmers. Methods Mol Biol 2000, 132:365-386.

27. Kuru T, Janusz N, Gadisa E, Gedamu L, Aseffa A: Leishmania aethiopica: development of specific and sensitive PCR diagnostic test. Exp Parasitol 2011, 128(4):391-395.

28. Macedo AM, Pimenta JR, Aguiar RS, Melo Al, Chiari E, Zingales B, Pena SD, Oliveira RP: Usefulness of microsatellite typing in population genetic studies of Trypanosoma cruzi. Mem Inst Oswaldo Cruz 2001, 96(3):407-413.

29. Ochsenreither S, Kuhls K, Schaar M, Presber W, Schönian G: Multilocus microsatellite typing as a new tool for discrimination of Leishmania infantum MON-1 strains. J Clin Microbiol 2006, 44(2):495-503.

30. Requena J, Chicharro C, Garcia L, Parrado R, Puerta C, Canavate C: Sequence analysis of the 3'-untranslated region of HSP70 (type I) genes in the genus Leishmania: its usefulness as a molecular marker for species identification. Parasites \& Vectors 2012, 5(1):87.

31. Gyapay G, Morissette J, Vignal A, Dib C, Fizames C, Millasseau P, Marc S, Bernardi G, Lathrop M, Weissenbach J: The 1993-94 Généthon human genetic linkage map. Nat Genet 1994, 7(2 Spec No):246-339.

32. Hanis CL, Boerwinkle E, Chakraborty R, Ellsworth DL, Concannon P, Stirling B, Morrison VA, Wapelhorst B, Spielman RS, Gogolin-Ewens KJ, et al: A genome-wide search for human non-insulin-dependent (type 2) diabetes genes reveals a major susceptibility locus on chromosome 2 . Nat Genet 1996, 13(2):161-166.

33. Fisher MC, Aanensen D, De Hoog S, Vanittanakom N: Multilocus microsatellite typing system for Penicillium marneffei reveals spatially structured populations. J Clin Microbiol 2004, 42(11):5065-5069.

doi:10.1186/1756-3305-6-160

Cite this article as: Kebede et al:: Multilocus microsatellite signature and identification of specific molecular markers for Leishmania aethiopica. Parasites \& Vectors 2013 6:160.

\section{Submit your next manuscript to BioMed Central and take full advantage of:}

- Convenient online submission

- Thorough peer review

- No space constraints or color figure charges

- Immediate publication on acceptance

- Inclusion in PubMed, CAS, Scopus and Google Scholar

- Research which is freely available for redistribution 